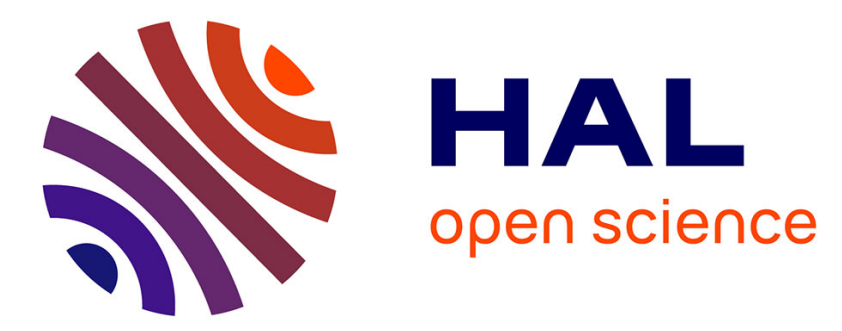

\title{
Quantum walks simulating non-commutative geometry in the Landau problem
}

F. Debbasch

\section{To cite this version:}

F. Debbasch. Quantum walks simulating non-commutative geometry in the Landau problem. J.Math.Phys., 2021, 62 (6), pp.062205. 10.1063/5.0030191 . hal-03288769

\section{HAL Id: hal-03288769 \\ https://hal.science/hal-03288769}

Submitted on 20 Sep 2021

HAL is a multi-disciplinary open access archive for the deposit and dissemination of scientific research documents, whether they are published or not. The documents may come from teaching and research institutions in France or abroad, or from public or private research centers.
L'archive ouverte pluridisciplinaire HAL, est destinée au dépôt et à la diffusion de documents scientifiques de niveau recherche, publiés ou non, émanant des établissements d'enseignement et de recherche français ou étrangers, des laboratoires publics ou privés. 


\section{Quantum walks simulating non- commutative geometry in the Landau problem}

Cite as: J. Math. Phys. 62, 062205 (2021); https://doi.org/10.1063/5.0030191

Submitted: 20 September 2020 . Accepted: 21 May 2021 . Published Online: 18 June 2021

D. Febbasch

\section{ARTICLES YOU MAY BE INTERESTED IN}

Nonlinear wave equation in a cosmological Kaluza Klein spacetime

Journal of Mathematical Physics 62, 062504 (2021); https://doi.org/10.1063/5.0046459

On the modified logarithmic Sobolev inequality for the heat-bath dynamics for 1D systems Journal of Mathematical Physics 62, 061901 (2021); https://doi.org/10.1063/1.5142186

Stationary scattering theory for repulsive Hamiltonians

Journal of Mathematical Physics 62, 061504 (2021); https://doi.org/10.1063/5.0031703

\section{Sign up for Alerts}

Journal of

Mathematical Physics

Receive the latest research updates 


\title{
Quantum walks simulating non-commutative geometry in the Landau problem
}

\author{
Cite as: J. Math. Phys. 62, 062205 (2021); doi: 10.1063/5.0030191 \\ Submitted: 20 September 2020 - Accepted: 21 May 2021 • \\ Published Online: 18 June 2021
}

\section{F. Debbasch}

\section{AFFILIATIONS}

Sorbonne Université, Observatoire de Paris, Université PSL, CNRS, LERMA, F-75005 Paris, France

a) Author to whom correspondence should be addressed: fabrice.debbasch@gmail.com

\begin{abstract}
Non-Commutative Geometry (NCG) is considered in the context of a charged particle moving in a uniform magnetic field. The classical and quantum mechanical treatments are revisited, and a new marker of NCG is introduced. This marker is then used to investigate NCG in magnetic Quantum Walks (QWs). It is proven that these walks exhibit NCG at and near the continuum limit. For the purely discrete regime, two illustrative walks of different complexities are studied in full detail. The most complex walk does exhibit NCG, but the simplest, most degenerate one does not. Thus, NCG can be simulated by QWs, not only in the continuum limit but also in the purely discrete regime.
\end{abstract}

Published under an exclusive license by AIP Publishing. https://doi.org/10.1063/5.0030191

\section{INTRODUCTION}

Quantum Walks (QWs) are automata defined on graphs and lattices. They were first considered by Feynman in studying possible discretizations for the Dirac path integral. ${ }^{1,2}$ They were later introduced in a systematic way by Aharonov et al. ${ }^{3}$ and Meyer. ${ }^{4}$ QWs are useful in quantum information and algorithmic development ${ }^{5-7}$ because they are a universal computational primitive. ${ }^{8,9}$ They are also important for quantum simulation, ${ }^{10}$ and they have been realized experimentally in a number of ways, ${ }^{7}$ which include cold atoms, ${ }^{11}$ photonic systems, ${ }^{12,13}$ and trapped ions. ${ }^{14}$

There is now growing literature on the geometrical aspects of QWs. QWs can indeed be used to simulate Dirac fermions interacting with arbitrary Yang-Mills gauge fields ${ }^{15,16}$ and arbitrary relativistic gravitational fields. ${ }^{17-21}$ In particular, exact discrete gauge invariant Yang-Mills field strengths can be built with QWs, ${ }^{22}$ and one can even construct a discrete counterpart to the Riemann curvature tensor. ${ }^{23}$ In addition, discussions of the Lorentz invariance of QWs can be found in Refs. 24-27 and the symplectic discrete geometry behind some QWs has been presented in Ref. 26.

The interface between QWs and geometry has also been explored on QWs defined on various structures with interesting geometries, be it different types of lattices ${ }^{28-32}$ or Cayley graphs, ${ }^{33-36}$ including Cayley graphs of non-Abelian groups. In several cases, a continuum limit exists and coincides with physics or mathematics on known differential manifolds. Some of these walks can also be coupled to gauge fields.

Now, what about the interface between QWs and Non-Commutative Geometry (NCG)? NCG, which was introduced more than seventy years ago, ${ }^{37}$ has become an important part of modern physics, arising in contexts as diverse as the quantum Hall effect, ${ }^{38}$ fluid dynamics, ${ }^{39,40}$ string theory, and M-theory. ${ }^{41}$ Yet, there is no real discussion in the literature of a possible quantum simulation of NCG. The interesting articles cited above that deal with QWs defined on non-Abelian Cayley graphs do come close to the topic, but they do not deal explicitly with NCG defined in the usual manner, i.e., through the non-vanishing of the commutator between two coordinates.

The virtual absence of NCG from the quantum simulation literature is all the more surprising because the simplest example of NCG in physics can be found in the so-called Landau problem, ${ }^{42-44}$ which describes the dynamics of a point particle (electron) in a uniform and constant magnetic field, and QWs have been proposed to simulate this problem, ${ }^{45}$ together with possible experimental realizations through cold atoms. ${ }^{46}$ 
The aim of the present article is to show that QWs can be used to perform a quantum simulation of NCG in the Landau problem. We start by revisiting magnetic field induced NCG, first in the context of classical mechanics and then in the context of non-relativistic spinless quantum mechanics. Addressing these relatively simple situations allows us to develop an intuition about NCG in the Landau problem, and we also set up the tools that will be useful in the rest of this article. We then switch to QWs, focusing on these so-called magnetic QWs, which can simulate the relativistic quantum Landau problem. We show that magnetic QWs at and near the continuum limit do realize NCG. We then explore the purely discrete regime through two relatively simple examples where all computations can be carried out at least seminumerically, if not analytically. The most complex walk does exhibit NCG, but the simplest, most degenerate one does not. Moreover, the discrete NCG, when it occurs, displays a non-linearity and a purely local (as opposed to global) character that the continuous NCG does not. We finally summarize and discuss all results in Sec. IV. The general conclusion is that QWs, both in their continuum and purely discrete regimes, can simulate NCG in the Landau problem.

\section{SPINLESS PARTICLE}

\section{A. Classical mechanics}

Consider the planar motion of a point charge $e$ of mass $m$ submitted to a constant and uniform magnetic field $\mathbf{B}$ perpendicular to the plane of motion. The equations of motion read

$$
\begin{aligned}
& \ddot{x}=\omega \dot{y}, \\
& \ddot{y}=-\omega \dot{x},
\end{aligned}
$$

where $x$ and $y$ are two orthonormal coordinates in the plane, $\omega=e B / m m$, and a derivation with respect to the time $t$ is designated by a dot. The complex velocity $V=\dot{x}+i \dot{y}$ obeys $\dot{V}=-i \omega V$, which leads to

$$
V(t)=V_{0} \exp (-i \omega t)
$$

where $V_{0}=v_{0 x}+i v_{0 y}$ is the initial complex velocity. Integrating the expression for $V(t)$ delivers

$$
X(t)=i \frac{V_{0}}{\omega} \exp (-i \omega t)+X_{c}
$$

where $X(t)=x(t)+i y(t)$ and $X_{c}=x_{c}+i y_{c}$ is a complex integration constant. Equation (3) leads immediately to

$$
\left|X(t)-X_{c}\right|^{2}=\left(x(t)-x_{c}\right)^{2}+\left(y(t)-y_{c}\right)^{2}=R^{2},
$$

with $R=\left|V_{0}\right| / \omega$. The variable $X(t)$ oscillates harmonically around its time-average $X_{c}$ and the trajectory of the particle is a circle of radius $R$ centered on $\left(x_{c}, y_{c}\right)$.

It is possible to consider that $X(t)$ is approximately equal to $X_{c}$ at all times if $\left|X(t)-X_{c}\right| /\left|X_{c}\right| \ll 1$, i.e., if the amplitude of the oscillations is very small compared to $X_{c}$. This translates into $\left|V_{0}\right| / \omega \ll\left|X_{c}\right|$.

The Lagrangian $L$ and the Hamiltonian $H$ for a particle moving in an electromagnetic field involve the vector potential $A$, ${ }^{47}$ so writing $L$ and $H$ makes it necessary to choose a gauge for $A$. We choose the so-called longitudinal gauge, where $A_{x}=0$ and $A_{y}=-B x$, and the Lagrangian then reads

$$
L(x, y, \dot{x}, \dot{y})=\frac{m}{2}\left(\dot{x}^{2}+\dot{y}^{2}\right)+e B x \dot{y}
$$

The momentum conjugate to $x$ and $y$ are $p_{x}=m \dot{x}$ and $p_{y}=m \dot{y}+e B x$, respectively, and the Hamiltonian reads

$$
H\left(x, y, p_{x}, p_{y}\right)=\frac{1}{2 m}\left(p_{x}^{2}+\left(p_{y}-e B x\right)^{2}\right)
$$

As shown previously, the two-degrees of freedom Hamiltonian system is integrable. It thus admits at least two independent first integrals. All trajectories are actually bounded and periodic (as opposed to pseudo-periodic), so there are actually three independent first integrals. The first of these is obvious: it is the time-independent Hamiltonian, which represents the conserved kinetic energy of the particle. The second integral is nearly as obvious, but its interpretation is not. Since $L$ (and $H$ ) do not depend explicitly on $y$, the momentum $p_{y}$ is conserved. Using the integrated expressions for $V$ and $X$ given above shows that, on the motion, $p_{y}$ coincides with $e B x_{c}=m \omega x_{c}$. The final first integral is $p_{x}+m \omega y$, which coincides on the motion with $m \omega y_{c}$.

Non-commutative geometry, i.e., non-commutation of $x$ and $y$, appears when one approximates the circular trajectory of the particle by its center, i.e., neglects the oscillations in the variable $X$. Consider indeed the Lagrangian $\tilde{L}$ defined by 


$$
\tilde{L}(x, y, \dot{x}, \dot{y})=\frac{m}{2} \dot{x}^{2}+e B x \dot{y},
$$

which can be obtained from $L$ by neglecting $\frac{m}{2} \dot{y}^{2}$ compared to $\frac{m}{2} \dot{x}^{2}$. The momentum $\tilde{p}_{x}$ is identical to $p_{x}=m \dot{x}$ but $\tilde{p}_{y}=e B x=m \omega x$. This relation is a first class constraint. It makes the $x$ variable proportional to $\tilde{p}_{y}$, which has a non-vanishing Poisson bracket with $y$. If one were to follow Dirac's procedure to quantize the dynamics of $\tilde{L}$, the first class constraint would have to be enforced on the physically admissible states, ensuring that, for these states, the operator $x$ is always proportional to the operator $\tilde{p}_{y}$, which does not commute with the operator $y$. The equations of motion derived from $\tilde{L}$ reveal how this non-commutative geometry relates to neglecting the spatial oscillations of the variable $X(t)$. The $y$-equation of motion derived from $\tilde{L}$ is $\dot{\tilde{p}}_{y}=0$, which implies $\dot{x}=0$, so $x=x_{c}$ and the $x$-equation of motion is $\dot{\tilde{p}}_{x}=e B \dot{y}$, which is equivalent to $m \ddot{x}=e B \dot{y}$. Since $x=x_{c}$, this implies $\dot{y}=0$, i.e., $y=y_{c}$.

Let us end this discussion by pointing out that the point $x_{c}+i y_{c}$ is actually the exact time average of $X(t)$. We will see in Sec. II B that NCG in the quantum mechanical treatment of the Landau problem can also appear in two different but related manners: one that relies on neglecting the width of a wave-function, which is the quantum mechanical equivalent of neglecting the classical amplitude of oscillations, and one that relies on an averaging and does not entail any approximation.

\section{B. Quantum mechanics}

Coordinates, momentum components, and Hamiltonian become the operators $\hat{x}, \hat{y}, \hat{p}_{x}, \hat{p}_{y}$, and $\hat{H}$. The Hamiltonian $\hat{H}$ commutes with $\hat{p}_{y}$ because $\hat{p}_{y}$ generates translations in the $y$ direction and $\hat{H}$ is independent of $\hat{y}$. We, therefore, search for eigenstates of both $\hat{H}$ and $\hat{p}_{y}$. As well known, the spectrum of $\hat{p}_{y}$ is the set of real numbers $\mathbb{R}$. For each real number $p$, let $\mathscr{H}_{p}$ be the subspace spanned by the eigenvectors of $\hat{p}_{y}$ associated with the eigenvalue $p$. On $\mathscr{H}_{p}$, the Hamiltonian operator coincides with $\hat{H}_{p}$ given by

$$
\hat{H}_{p}=\frac{1}{2 m} \hat{p}_{x}^{2}+\frac{m \omega^{2}}{2}(\hat{x}-\hat{x}(p))^{2},
$$

where $\omega=e B / m$ (as in Sec. II A) and $\hat{x}(p)$ is the operator that, in the $x$-representation, coincides with the multiplication by $x(p)=p /(e B)$ $=p /(m \omega)$. In other words, $\hat{x}(p)=\hat{p}_{y} /(m \omega)$.

The reduced Hamiltonian $\hat{H}_{p}$ generates the dynamics of an harmonic oscillator of mass $m$ and pulsation $\omega$, centered on $x(p)$. The energy eigenstates of $\hat{H}_{p}$ are non-degenerate, indexed by $n \in \mathbb{N}$, with eigenvalues $E_{n}=(n+1 / 2) \hbar \omega$ independent of $p$. The wave-function of the state $|n, p\rangle$ is the product of a Gaussian by a Hermite polynomial, both centered on $x(p)$. It is either symmetric (even $n$ ) or antisymmetric (uneven $n$ ) in $x-x(p)$ and has typical width $|x-x(p)| \sim((n+1 / 2) \hbar /(m \omega))^{1 / 2}=(n+1 / 2)^{1 / 2} a$, where the length $a=(\hbar /(m \omega))^{1 / 2}$ is also independent of $p$. The eigenvalue $p$ of $p_{y}$ thus enters the eigenstate of $\hat{H}_{p}$ only through the shift $x(p)$.

In any given state, the coordinate $x$ can be viewed as a random variable with the probability law given by Born's law. Since the wavefunctions of the states $|n, p\rangle$ are either symmetric or antisymmetric in $x-x(p)$, the average value of $x$ in any eigenstate $|n, p\rangle$ is identical to $x(p)$. By definition, the average of $x$ in any given state coincides with the expectation value of the operator $\hat{x}$ in that state. Thus,

$$
\langle n, p|\hat{x}| n, p\rangle=x(p) .
$$

Since the states $|n, p\rangle$ form a basis of the Hilbert space, an arbitrary operator can be defined by its action on these states. We define an average position operator $\langle\hat{x}\rangle$ by

$$
\langle\hat{x}\rangle|n, p\rangle=\langle n, p|\hat{x}| n, p\rangle|n, p\rangle .
$$

By Eq. (9), this average position operator coincides with the operator $\hat{x}(p)$ introduced above [see Eq. (8)], which is itself proportional to the operator $\hat{p}_{y}$, with multiplicative factor $1 /(m \omega)$. Thus, averaging or taking the expectation value delivers non-commutative geometry in the sense that $[\hat{y},\langle\hat{x}\rangle]=i \hbar /(m \omega)$, as can be checked directly on the wave-functions. This is true for all values of the parameters.

Non-commutative geometry also appears without averaging, but at the cost of an approximation. Suppose that the width $|x-x(p)|$ of the wave-function is much smaller than $x(p)$, i.e., $|e B| \ll p^{2} /\left(\hbar(n+1 / 2)^{2}\right)$. The probability law for $x$ is then essentially a Dirac distribution on $x(p)$, and one gets $[\hat{y}, \hat{x}] \simeq i \hbar /(m \omega)$.

\section{QUANTUM WALKS}

\section{A. Magnetic quantum walks}

A discrete time QW simulating on a Cartesian space-time grid the $2 D$ motion of a particle immersed in a constant and uniform magnetic field has been proposed in Ref. 45 . Let $j \in \mathbb{N}$ be the discrete time and $(q, r) \in \mathbb{Z}^{2}$ be the discrete position on the $2 D$ grid perpendicular to the magnetic field $B$. The equations defining the walk read as 


$$
\begin{aligned}
\psi_{j+1, q, r}^{L} & =e^{2 i \alpha_{q}} c^{-}\left[c^{+} \psi_{j, q+1, r+1}^{L}+i s^{+} \psi_{j, q-1, r+1}^{R}\right]+i e^{-2 i \alpha_{q}} s^{-}\left[i s^{+} \psi_{j, q+1, r-1}^{L}+c^{+} \psi_{j, q-1, r-1}^{R}\right], \\
\psi_{j+1, q, r}^{R} & =i e^{2 i \alpha_{q}} s^{-}\left[c^{+} \psi_{j, q+1, r+1}^{L}+i s^{+} \psi_{j, q-1, r+1}^{R}\right]+e^{-2 i \alpha_{q}} c^{-}\left[i s^{+} \psi_{j, q+1, r-1}^{L}+c^{+} \psi_{j, q-1, r-1}^{R}\right],
\end{aligned}
$$

where $\psi^{L / R}$ are the two components of the "spinor;" $\alpha_{q}=\varepsilon^{2} e B q /(2 \hbar) ; c^{ \pm}=\cos \theta^{ \pm}$and $s^{ \pm}=\sin \theta^{ \pm}$, with $\theta^{ \pm}= \pm \pi / 4-\varepsilon m /(2 \hbar)$; and $\varepsilon$ is a real positive parameter of the walk. An interesting continuum limit can be obtained by defining $t_{j}=\varepsilon j, x_{q}=\varepsilon q$, and $y_{r}=\varepsilon r$, assuming analyticity in $\varepsilon$ and letting $\varepsilon$ tend to 0 . This delivers

$$
\begin{aligned}
& \hbar\left(\partial_{t}-\partial_{x}\right) \psi^{L}-i\left(\hbar \partial_{y}+i e B x-m\right) \psi^{R}=0, \\
& i\left(\hbar \partial_{y}+i e B x+m\right) \psi^{L}+\hbar\left(\partial_{t}+\partial_{x}\right) \psi^{R}=0,
\end{aligned}
$$

which is the Dirac equation describing the planar motion of a spin $1 / 2$ charge $e$ immersed in the magnetic field $B$ orthogonal to the plane of motion. The velocity of light $c$ is set to unity in the whole article.

To investigate if NCG appears, we search for stationary states of the QW (or of the Dirac dynamics) that are also eigenstates of the momentum operator $\hat{p}_{y}$. We thus write $\Psi_{j, q, r}^{p}=\Phi_{j, q} \exp \left(-i p \varepsilon^{2} r / \hbar\right)$ and obtain for $\Phi$ the following equations:

$$
\begin{aligned}
& \phi_{j+1, q}^{L}=e^{2 i \beta_{q}} c^{-}\left[c^{+} \phi_{j, q+1}^{L}+i s^{+} \phi_{j, q-1}^{R}\right]+i e^{-2 i \beta_{q}} s^{-}\left[i s^{+} \phi_{j, q+1}^{L}+c^{+} \phi_{j, q-1}^{R}\right], \\
& \phi_{j+1, q}^{R}=i e^{2 i \beta_{q}} s^{-}\left[c^{+} \phi_{j, q+1}^{L}+i s^{+} \phi_{j, q-1}^{R}\right]+e^{-2 i \beta_{q}} c^{-}\left[i s^{+} \phi_{j, q+1}^{L}+c^{+} \phi_{j, q-1}^{R}\right],
\end{aligned}
$$

with $\beta_{q}=\alpha_{q}-\varepsilon^{2} p /(2 h)=\varepsilon e B\left(x_{q}-x(p)\right) /(2 h)$. The evolution of $\Phi$ is thus a $p$-dependent QW on the line. Nevertheless, for readability purposes, the retained notation does not make the dependence of $\beta$ and $\Phi$ on $p$ explicit. At the continuum limit, the equations for $\Phi$ read

$$
\begin{aligned}
\hbar\left(\partial_{t}-\partial_{x}\right) \phi^{L}+(e B(x-x(p))+i m) \phi^{R} & =0 \\
(-e B(x-x(p))+i m) \phi^{L}+\hbar\left(\partial_{t}+\partial_{x}\right) \phi^{R} & =0 .
\end{aligned}
$$

The expression of $\beta_{q}$ given above and the appearance of the difference $x-x(p)$ in the continuum limit Dirac equation suggest that NCG is also present in the discrete time QW and, in particular, in the Dirac equation. To prove or disprove that intuition, one needs to look at the energy-eigenstates of the dynamics. This can be done exactly at the continuum limit and at first order around this limit, but there does not seem to be a general expression for these states, valid for all values of the walk parameters. We therefore start by a general presentation of NCG at the continuum limit and at first perturbation order around this limit and then switch to examples that illustrate possible behaviors of the QW in the purely discrete regime.

\section{B. NCG at and near the continuum limit}

The energy eigenstates for $\Phi$ have been computed in Ref. 15 at first order in the perturbation parameter $\varepsilon$ around the continuum limit $\varepsilon=0$. After a change of orthonormal basis in spin space, the components of these eigenstates are, at zeroth and first order in $\varepsilon$, even or uneven functions of $x-x(p)$, so the average of the variable $x$ in any state $|n, p\rangle$ is identical to $x(p)$. Thus, as for the spinless non-relativistic quantum mechanical problem, $[\hat{y},\langle\hat{x}\rangle]=i \hbar /(m \omega)$ at zeroth and first order in $\varepsilon$, i.e., at and near the continuum limit.

In the new spin basis, the typical spatial extensions of the components of the eigenstate labeled by $n$ are $((n+1 / 2) a)^{1 / 2}$ and $((n-1 / 2) a)^{1 / 2}$, where $a=\hbar \omega$. Both these extensions are much smaller than $|x(p)|$ if $|e B| \ll p^{2} /\left(\hbar(n+1 / 2)^{2}\right)$, which is the same condition as the one found in the quantum mechanical treatment. If this is realized, then $[\hat{y}, \hat{x}] \simeq i \hbar /(m \omega)$ and one gets again NCG.

Thus, at and near the continuum limit, QWs essentially behave as solutions of the spinless Schrödinger equation, as far as NCG is concerned. This may seem surprising for a variety of reasons. First, one might have expected differences at the continuum limit, i.e., for $\varepsilon=0$, because the Dirac equation is mathematically very different from the spinless Schrödinger equation. In particular, the Dirac Hamiltonian is quite different from the Schrödinger Hamiltonian and one might have expected that the modifications of the energy eigenstates due to the spin 1/2 of Dirac fermions might, in turn, modify the magnetic field induced NCG. Second, the ultimate reason why NCG is not modified at first order in $\varepsilon$ is that the energy and momentum eigenstates are the same at first and zeroth orders in $\varepsilon$. This fact is not trivial and rather surprising in itself because, contrary to the eigenstates, the eigenenergies actually do contain a non-vanishing first order contribution. ${ }^{15}$ To sum up, taking spin and special relativity into account does not bring anything new to the NCG discussion, even at first order in $\varepsilon$. Things however change when one considers NCG in the purely discrete regime. 


\section{Purely discrete case}

We now consider situations where $\varepsilon=1$ and $e B / \hbar=2 \pi / N$, where $N$ is a positive integer. The Hilbert space is then made of $N$-periodic functions on the $q$-axis and is therefore of dimension $2 N$. Assuming the square grid infinite in the $r$-direction, the momentum $\bar{p}=p / \hbar$ lies in an interval of length $2 \pi$, say, $(0,2 \pi)$. Suppose now one wants to investigate NCG in QWs using the same type of approximation as the one used in the quantum mechanical treatment. One would have to consider walks whose extension in the $q$-variable is much smaller than $\bar{p}$. However, the extension of a walk cannot be smaller than the grid step, which is unity, and $\bar{p}$ is never much larger than unity because it is in $(0,2 \pi)$. Thus, the approximation used in the quantum mechanical treatment never applies to discrete automata, such as QWs. However, the other way to exhibit NCG, which is based on averaging, does work, as can be seen in Example 1.

\section{Example 1}

Consider a walk with vanishing mass $m$, so $\theta_{+}=-\theta_{-}=\pi / 4$. The equations of motion simplify into

$$
\begin{aligned}
\phi_{j+1, q}^{L} & =\cos \left(2 \beta_{q}\right) \phi_{j, q+1}^{L}+\sin \left(2 \beta_{q}\right) \phi_{j, q-1}^{R}, \\
\phi_{j+1, q}^{R} & =-\sin \left(2 \beta_{q}\right) \phi_{j, q+1}^{L}+\cos \left(2 \beta_{q}\right) \phi_{j, q-1}^{R} .
\end{aligned}
$$

The coefficients in these equations are $2 \pi$-periodic in $\bar{p}$

Let us now specialize to the first non-trivial case, i.e., $N=3$ and choose $\{0,1,2\}$ as the spatial periodicity set. The time-independent eigenvectors of the dynamics satisfy

$$
\begin{aligned}
& \lambda \phi_{0}^{L}=\cos \left(2 \beta_{0}\right) \phi_{1}^{L}+\sin \left(2 \beta_{0}\right) \phi_{2}^{R}, \\
& \lambda \phi_{0}^{R}=-\sin \left(2 \beta_{0}\right) \phi_{1}^{L}+\cos \left(2 \beta_{0}\right) \phi_{2}^{R}, \\
& \lambda \phi_{1}^{L}=\cos \left(2 \beta_{1}\right) \phi_{2}^{L}+\sin \left(2 \beta_{1}\right) \phi_{0}^{R}, \\
& \lambda \phi_{1}^{R}=-\sin \left(2 \beta_{1}\right) \phi_{2}^{L}+\cos \left(2 \beta_{1}\right) \phi_{0}^{R}, \\
& \lambda \phi_{2}^{L}=\cos \left(2 \beta_{2}\right) \phi_{0}^{L}+\sin \left(2 \beta_{2}\right) \phi_{1}^{R}, \\
& \lambda \phi_{2}^{R}=-\sin \left(2 \beta_{2}\right) \phi_{0}^{L}+\cos \left(2 \beta_{2}\right) \phi_{1}^{R} .
\end{aligned}
$$

A direct computation reveals that the characteristic equation obeyed by $\lambda$ is

$$
\lambda^{6}-\frac{3}{4} \lambda^{4}-\frac{\cos (3 \bar{p})}{2} \lambda^{3}-\frac{3}{4} \lambda^{2}+1=0 .
$$

The spectrum is thus $2 \pi / 3$-periodic in $\bar{p}$. On the period $(0,2 \pi / 3)$, the spectrum is also symmetric with respect to $\bar{p}=\pi / 3$. The dynamics is unitary, so all eigenvalues lie on the unit circle. Since all coefficients in the characteristic equation are real, the solutions appear as couples of complex conjugates $[\exp (i \theta), \exp (-i \theta)]$ with, say, $\theta \in(0, \pi)$. A numerical computation shows that the characteristic equation admits generically six distinct solutions on the unit circle, except for $\bar{p}=0, \pi / 3,2 \pi / 3,4 \pi / 3$, where the equation only admits five distinct solutions. Finally, the eigenvalue/vector problem is invariant under the following two transformations:

$$
\begin{aligned}
& p \rightarrow p+2 \pi / 3, \Phi_{0} \rightarrow \Phi_{1}, \Phi_{1} \rightarrow \Phi_{2}, \Phi_{2} \rightarrow \Phi_{0}, \\
& p \rightarrow p+4 \pi / 3, \Phi_{0} \rightarrow \Phi_{2}, \Phi_{1} \rightarrow \Phi_{0}, \Phi_{2} \rightarrow \Phi_{1} .
\end{aligned}
$$

We now focus, for each value of $\bar{p}$, on the eigenvalue corresponding to the smallest positive value $\theta_{s}$ of the angle $\theta$, which is the discrete equivalent of the lowest positive energy. Figure 1 presents $\theta_{S}$ as a function of $\bar{p}$ on the period $(0,2 \pi / 3)$. Each of the corresponding normalized eigenvectors generates a probability law on the set $\{0,1,2\}$, and this law can be used to define and compute the average $\langle q\rangle$ as a function of $\bar{p}$. This function $A$ is $2 \pi$-periodic, as are the coefficients of the equations obeyed by $\Phi$. It is plotted in Fig. 2 .

NCG follows from the above results by the following reasoning. Let $f$ be an arbitrary, possibly $q$-dependent function of $r$. Its discrete Fourier transform

$$
\hat{f}(\bar{p})=\frac{1}{\sqrt{2 \pi}} \sum_{r} f_{r} \exp (-i \bar{p} r)
$$

is defined for $\hat{p} \in(0,2 \pi)$, and the inverse transform reads

$$
f_{r}=\frac{1}{\sqrt{2 \pi}} \int_{0}^{2 \pi} \hat{f}(\bar{p}) \exp (i \bar{p} r) d \bar{p}
$$




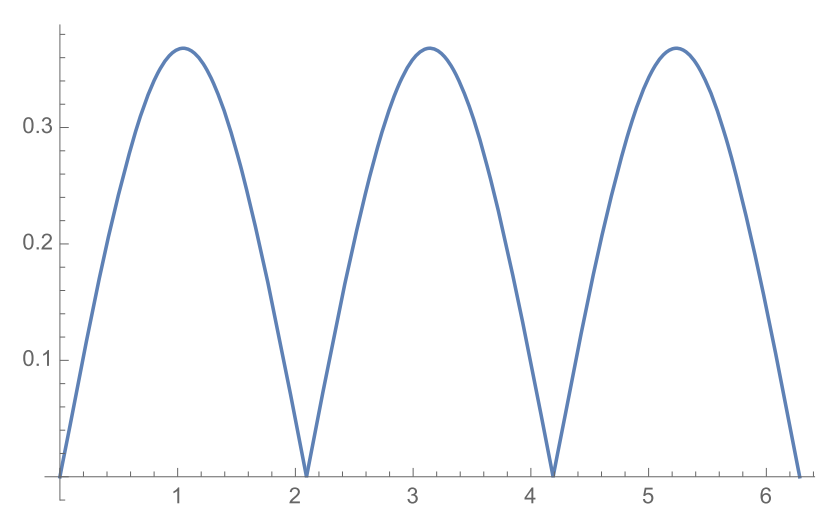

FIG. 1. Example 1. Angle $\theta_{S}$ as a function of the reduced momentum variable $\bar{p}$.

It follows from the second of these equations that

$$
\begin{aligned}
r f_{r} & =\frac{1}{\sqrt{2 \pi}} \int_{0}^{2 \pi} \hat{f}(\bar{p}) r \exp (i \bar{p} r) d \bar{p} \\
& =\frac{1}{\sqrt{2 \pi}} \int_{0}^{2 \pi} \hat{f}(\bar{p})\left(-i \partial_{\bar{p}} \exp (i \bar{p} r)\right) d \bar{p} \\
& =\frac{1}{\sqrt{2 \pi}} \int_{0}^{2 \pi}\left(i \frac{d \hat{f}}{d \bar{p}}\right) \exp (i \bar{p} r) d \bar{p}
\end{aligned}
$$

where an integration by parts and the identity $\hat{f}(2 \pi) \exp (i 2 \pi r)=\hat{f}(0) \exp (i 0 \times r)$ have been used to obtain the last equation. This shows that the operator $\hat{r}$ is identical to the operator $i d / d \bar{p}$ and that therefore $[\hat{r}, \hat{p}]=i$ or, if one prefers, $[\hat{r}, \hat{p}]=i \hbar$. Using $\langle q\rangle=A(\bar{p})$ then delivers

$$
[\hat{r},\langle\hat{q}\rangle]=i A^{\prime}(\hat{\bar{p}})
$$

As seen in Fig. 2, the function $A$ and thus its derivative can be inverted locally, but not globally. Thus, locally, (22) transcribes into an equation of the form

$$
[\hat{r},\langle\hat{q}\rangle]=i B(\langle\hat{q}\rangle)
$$

where $B$ is a non-linear function.

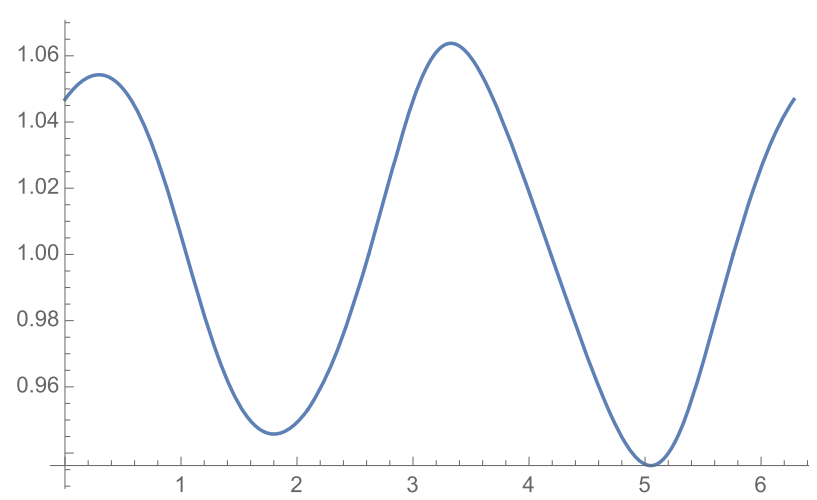

FIG. 2. Example 1. Average position $\langle q\rangle$ as a function of the reduced momentum variable $\bar{p}$. 


\section{Example 2}

Consider now a walk with $m / \hbar=\pi / 2$ so that $\theta_{+}=\pi / 2$ and $\theta_{-}=0$. The equations of motion for $\phi$ read

$$
\begin{aligned}
& \phi_{j+1, q}^{L}=i e^{2 i \beta_{q}} \phi_{j, q-1}^{R}, \\
& \phi_{j+1, q}^{R}=i e^{-2 i \beta_{q}} \phi_{j, q+1}^{L} .
\end{aligned}
$$

Choosing as the spatial periodicity set $\{0,1, N-1\}$, the time-independent eigenvectors of the dynamics satisfy

$$
\begin{aligned}
\lambda \phi_{0}^{L} & =i e^{2 i \beta_{0}} \phi_{N-1}^{R}, \\
\lambda \phi_{N-1}^{R} & =i e^{-2 i \beta_{N-1}} \phi_{0}^{L}, \\
\lambda \phi_{1}^{L} & =i e^{2 i \beta_{1}} \phi_{0}^{R}, \\
\lambda \phi_{0}^{R} & =i e^{-2 i \beta_{0}} \phi_{1}^{L}, \\
\vdots & \\
\lambda \phi_{N-1}^{L} & =i e^{2 i \beta_{N-1}} \phi_{N-2}^{R}, \\
\lambda \phi_{N-2}^{R} & =i e^{-2 i \beta_{N-2}} \phi_{N-1}^{L},
\end{aligned}
$$

where $\lambda$ is the associated eigenvalue. This system of $2 N$ equations splits into $N$ independent systems of two equations, and each system of two equations admits the same eigenvalues $\lambda_{ \pm}= \pm e^{i \pi / N}$. There are therefore only two energy levels $E_{ \pm}=\hbar \pi(1 / N+1 / 2 \pm 1 / 2)$, which are independent of $p$, but each level is $N$ times degenerate. Each of the eigenvectors is localized on a couple of points $(q-1, q)$ with the convention that $q-1=N-1$ for $q=0$. The only non-vanishing components of the normalized eigenvectors localized at $(q-1, q)$ are

$$
\begin{aligned}
\phi_{q}^{L} & =\frac{1}{\sqrt{2}}, \\
\phi_{q-1}^{R} & = \pm \frac{i}{\sqrt{2}} e^{i p / \hbar} e^{-i \pi(1+2(q-1)) / N}
\end{aligned}
$$

with $q-1=N-1$ if $q=0$. The eigenvectors do depend on the momentum $p$, but the associated probability densities do not. Thus, $p$ has no influence of the probability law for the position of the walker, and non-commutative geometry in the sense of the Secs. II, III A, III B, and III C 1 is not realized for these walks.

\section{DISCUSSION}

We have shown that NCG can be simulated with QWs. We have focused on the Landau problem, which describes the dynamics of a point particle in a uniform magnetic field. We have first revisited this problem in the contexts of classical mechanics and of spinless nonrelativistic quantum mechanics. We have then switched to so-called magnetic QWs, which model the relativistic dynamics of a spin $1 / 2$ quantum particle immersed in a magnetic field. We have shown that NCG is always realized in magnetic DQWs at and near the continuum limit. NCG can also be realized in the purely discrete regime if the structure of the walk is rich enough. To show that NCG does not arise for all walks in the discrete regime, we have finally studied a specific, highly degenerate magnetic walk where NCG does not occur. The general conclusion is that NCG can be simulated with QWs, for example, through quantum optics experiments. Let us now discuss all these results.

The treatment of NCG in the classical and quantum mechanical Landau problem proposed in this article differs from the existing ones in several aspects. It has been known for a long time that NCG can arise through an approximation in the classical and quantum mechanical Landau problem, but previous presentations do not exhibit a dimensionless parameter controlling this approximation. We have identified this parameter and given its physical interpretation. As a consequence, we have shown that, at a fixed value of the integer $n$, which indices the oscillatory Landau levels, NCG arises for small enough values of the magnetic field, and not large values, as has been sometimes previously asserted based on heuristic reasoning. Note that the only previous non-heuristic derivation of NCG in the classical and quantum mechanical Landau problem is based on restricting the Hilbert space allowed to the particle and this procedure does not involve a dimensionless infinitesimal, i.e., it is a formal procedure that cannot be interpreted in usual physical terms as the consequence of neglecting a certain quantity with respect to another one having the same physical dimension.

We have also shown that NCG arises in the classical and quantum mechanical Landau problem independently of any approximation if one considers the average position of the particle. This comes from the fact that, in all classical solutions and in all quantum mechanical energy eigenstates, the average of one coordinate, say, $x$, is proportional to the momentum associated with the other coordinate $y$. This new result is important for two reasons: first, because it shows that NCG is intrinsic to the Landau problem and is not an approximate property of the dynamics valid only for small (or large) enough values of the magnetic field and, second, because it is the approach in terms of the average position operator that can be extended to encompass discrete automata, such as QWs. 
The extension to QWs at or near the continuum limit deserves only a few comments. The limit itself and its first order perturbations have already been studied in depth in Ref. 15. The continuum limit obeys a Dirac equation. This equation can be reduced to the dynamics of two coupled harmonic oscillators and the components of the energy eigenstates are therefore very similar to the energy eigenstates of the quantum mechanical Landau problem. The discussion of NCG geometry therefore proceeds in a similar way. Considering first order perturbations around that continuum limit does not drastically change the picture. In particular, the average position operator of one coordinate is still proportional to the momentum of the other coordinate.

Things however do change a lot when one considers magnetic QWs away from their continuum limit. The general analytical determination of the energy eigenstates does not seem feasible. We have therefore concentrated on two examples that correspond to the values of the mass for which the equations of the walk simplify. The first example corresponds to a vanishing mass and is clearly the less degenerate of the two. In that case, NCG exists in the discrete regime, but it is much more complex than the NCG envisaged in Secs. II, III A, and III B. Indeed, the average of one coordinate, say, $x$, is again a function of the momentum $p$ associated with the other coordinate $y$, but this function is neither linear nor globally invertible. The commutator of $\langle\hat{x}\rangle$ and $\hat{y}$ is therefore not constant, but a function of $\hat{p}$, and this function can be inverted and written in terms of $\langle\hat{x}\rangle$, but only locally. The NCG that emerges is thus quite different from the simplest one encountered in the continuous case. This new NCG is both discrete and non-linear. It is controlled by the variable $\hat{p}$, which can be expressed in terms of $\langle\hat{x}\rangle$ only locally.

The second example is engineered to make the equations of motion of the walk as degenerate as possible. Because of the degeneracy, the wave-functions of the energy eigenstates depend exponentially on $p$, so the associated densities actually do not depend on $p$. It follows that the expectation value $\langle\hat{x}\rangle$ does not depend on $\hat{p}$ and there is no NCG is this degenerate case.

Evidently, this work should not be regarded as a proof that arbitrary NCG can be simulated with QWs. NCG exists in many incarnations and a lot of different contexts. In particular, models that view space-time geometry as fundamentally non-commutative have not been dealt with in this article, and simulating them with quantum automata, if possible, would require a much broader analysis. This article deals with what is possibly the simplest occurence of NCG in physics and, in simulating this kind of NCG with QWs, highlights the possible interplay between NCG and quantum automata, thus hopefully stimulating future research on the topic.

Let us now conclude by mentioning a few possible extensions to this work. One should first concentrate on the same family of magnetic walks and determine which walks generate NCG. The examples worked out in this article suggest that NCG will appear in all walks except the most degenerate ones, but confirming this statement and investigating exactly what the NCG looks like for various values of the parameters, i.e., how the non-vanishing commutator depends on the impulse variable, will clearly prove instructing. One should then consider other magnetic and electromagnetic walks, be they defined on the same grid, but with other unitaries, or be they defined on other grids, even possibly on graphs. For example, can QWs defined on Cayley graphs generate NCG and does this NCG correlate with the non-commutativity of the underlying group? As mentioned above, exploring how NCG appears in QWs simulating other physics than electromagnetism, notably in gauge theories and in quantum gravity, should also prove worthwhile. For example, the results presented in this article suggest that NCG also exists in other discrete models, such as LGTs. If a discrete NCG does arise in LGTs and how it differs from the discrete NCG of QWs remain to be determined. Finally, the occurence of NCG in quantum walks, which are a universal computational primitive, makes one wonder about the consequences of NCG in quantum algorithmics.

\section{DATA AVAILABILITY}

Data sharing is not applicable to this article as no new data were created or analyzed in this study.

\section{REFERENCES}

${ }^{1}$ R. P. Feynman and A. R. Hibbs, Quantum Mechanics and Path Integrals (McGraw-Hill Book Company, 1965).

${ }^{2}$ S. S. Schweber, Rev. Mod. Phys. 58, 449 (1986).

${ }^{3}$ Y. Aharonov, L. Davidovich, and N. Zagury, Phys. Rev. A 48, 1687 (1993).

${ }^{4}$ D. A. Meyer, J. Stat. Phys. 85, 551 (1996).

${ }^{5}$ A. Ambainis, SIAM J. Comput. 37, 210 (2007).

${ }^{6}$ F. Magniez, A. Nayak, J. Roland, and M. Santha, SIAM J. Comput. 40, 142 (2011).

${ }^{7}$ K. Manouchehri and J. B. Wang, Physical Implementation of Quantum Walks (Springer, 2014).

${ }^{8}$ A. M. Childs, Phys. Rev. Lett. 102, 180501 (2009).

${ }^{9}$ N. Lovett, S. Cooper, M. Everitt, M. Trevers, and V. Kendon, Phys. Rev. A 81, 042330 (2010).

${ }^{10}$ R. Gerritsma, G. Kirchmair, F. Zähringer, E. Solano, R. Blatt, and C. F. Roos, Nature 463, 68 (2010).

${ }^{11}$ M. Karski, L. Förster, J.-M. Choi, A. Steffen, W. Alt, D. Meschede, and A. Widera, Science 325, 174 (2009).

${ }^{12}$ A. Peruzzo, M. Lobino, J. C. F. Matthews, N. Matsuda, A. Politi, K. Poulios, X.-Q. Zhou, Y. Lahini, N. Ismail, K. Wörhoff, Y. Bromberg, Y. Silberberg, M. G. Thompson, and J. L. Obrien, Science 329, 1500 (2010).

${ }^{13}$ A. Schreiber, K. N. Cassemiro, V. Potocek, A. Gabris, P. Mosley, E. Andersson, I. Jex, and C. Silberhorn, Phys. Rev. Lett. 104, 050502 (2010).

${ }^{14}$ C. Huerta Alderete, S. Singh, N. H. Nguyen, D. Zhu, R. Balu, C. Monroe, C. M. Chandrashekar, and N. M. Linke, Nat. Commun. 11, 3720 (2020).

${ }^{15}$ P. Arnault and F. Debbasch, Phys. Rev. A 93, 052301 (2016).

${ }^{16}$ I. Márquez, P. Arnault, G. Di Molfetta, and A. Pérez, Phys. Rev. A 98, 032333 (2018).

${ }^{17}$ G. Di Molfetta, M. Brachet, and F. Debbasch, Phys. Rev. A 88, 042301 (2013). 
${ }^{18}$ G. Di Molfetta, M. Brachet, and F. Debbasch, Physica A 397, 157 (2014).

${ }^{19}$ P. Arrighi, S. Facchini, and M. Forets, Quantum Inf. Process. 15, 3467 (2016).

${ }^{20}$ P. Arrighi and S. Facchini, Quantum Inf. Comput. 17, 810 (2017).

${ }^{21}$ P. Arnault and F. Debbasch, Ann. Phys. 383, 645 (2017).

${ }^{22}$ P. Arnault, G. Di Molfetta, M. Brachet, and F. Debbasch, Phys. Rev. A 94, 012335 (2016).

${ }^{23}$ F. Debbasch, Condens. Matter 4, 40 (2019).

${ }^{24}$ P. Arrighi, S. Facchini, and M. Forets, New J. Phys. 16, 093007 (2014).

${ }^{25}$ A. Bisio, G. M. D'Ariano, and P. Perinotti, Found. Phys. 47, 1065 (2017).

${ }^{26}$ F. Debbasch, Ann. Phys. 405, 340 (2019).

${ }^{27}$ L. Apadula, A. Bisio, G. M. D’Ariano, and P. Perinotti, J. Math. Phys. 61, 082202 (2020).

${ }^{28}$ L. Bru, G. de Valcárcel, G. Di Molfetta, A. Pérez, E. Roldán, and F. Silva, Phys. Rev. A 94, 032328 (2016).

${ }^{29}$ G. Jay, F. Debbasch, and J. B. Wang, Phys. Rev. A 99, 032113 (2019).

${ }^{30}$ I. Boettcher, P. Bienias, R. Belyansky, A. Kollár, and A. Gorshkov, Phys. Rev. A 102, 032208 (2019).

${ }^{31}$ G. Jay, F. Debbasch, and J. Wang, Quantum Inf. Process. 19, 422 (2020).

${ }^{32}$ G. Jay, F. Arnault, and P. Debbasch, arXiv:2007.06211 (2021).

${ }^{33}$ O. L. Acevedo and T. Gobron, J. Phys. A: Math. Gen. 39, 585 (2006).

${ }^{34}$ A. Bisio, G. D'Ariano, M. Erba, P. Perinotti, and A. Tosini, Phys. Rev. A 93, 062334 (2016).

${ }^{35}$ G. D’Ariano, M. Erba, P. Perinotti, and A. Tosini, J. Phys. A: Math. Theor. 50, 035301 (2016).

${ }^{36}$ G. D’Ariano, M. Erba, and P. Perinotti, Phys. Rev. A 96, 062101 (2017).

${ }^{37}$ H. S. Snyder, Phys. Rev. 71, 38 (1947).

${ }^{38}$ J. Belissard, A. van Elst, and H. Schulz-Baldes, J. Math. Phys. 35, 5373 (1994).

${ }^{39}$ S. Boatto and D. Crowdy, Encyclopedia of Mathematical Physics (Academic Press, 2006), pp. 66-79.

${ }^{40}$ P. Das and S. Ghosh, Eur. Phys. J. C 76, 627 (2016).

${ }^{41}$ N. Seiberg and E. Witten, J. High Energy Phys. 1999, 032.

${ }^{42}$ R. Jackiw, Nucl. Phys. B, Proc. Suppl. 108, 30 (2002).

${ }^{43}$ G. Magro, arXiv:quant-ph/0302001.

${ }^{44}$ K. Andrzejewski and P. Maslanka, Phys. Rev. D 70, 107701 (2004).

${ }^{45}$ P. Arnault and F. Debbasch, Physica A 443, 179 (2016).

${ }^{46}$ M. Sajid, J. K. Asbóth, D. Meschede, R. F. Werner, and A. Alberti, Phys. Rev. B 99, 214303 (2019).

${ }^{47}$ L. D. Landau and E. Lifshitz, The Classical Theory of Fields (Butterworth-Heinemann, 1975). 